This item was submitted to Loughborough's Research Repository by the author.

Items in Figshare are protected by copyright, with all rights reserved, unless otherwise indicated.

\title{
Fission of a longitudinal strain solitary wave in a delaminated bar
}

PLEASE CITE THE PUBLISHED VERSION

http://dx.doi.org/10.1103/PhysRevE.77.066603

\section{PUBLISHER}

(C) American Physical Society

VERSION

VoR (Version of Record)

LICENCE

CC BY-NC-ND 4.0

\section{REPOSITORY RECORD}

Khusnutdinova, Karima R., and Alexander M. Samsonov. 2019. "Fission of a Longitudinal Strain Solitary Wave in a Delaminated Bar". figshare. https://hdl.handle.net/2134/15728. 


\title{
Fission of a longitudinal strain solitary wave in a delaminated bar
}

\author{
Karima R. Khusnutdinova* \\ Department of Mathematical Sciences, Loughborough University, Loughborough LE11 3TU, United Kingdom
}

Alexander M. Samsonov

Ioffe Physico-Technical Institute of the Russian Academy of Sciences, St. Petersburg 194021, Russia

(Received 24 October 2007; revised manuscript received 14 March 2008; published 10 June 2008)

\begin{abstract}
The aim of the paper is to show that splitting of a waveguide leads to fission of bulk solitons in solids. We study the dynamics of a longitudinal bulk solitary wave in a delaminated, symmetric layered elastic bar. First, we consider a two-layered bar and assume that there is a perfect interface when $x<0$ and complete debonding (splitting) when $x>0$, where the axis $O x$ is directed along the bar. We derive the so-called doubly dispersive equation (DDE) for a long nonlinear longitudinal bulk wave propagating in an elastic bar of rectangular cross section. We formulate the problem for a delaminated two-layered bar in terms of the DDE with piecewise constant coefficients, subject to continuity of longitudinal displacement and normal stress across the "jump" at $x=0$. We find the weakly nonlinear solution to the problem and consider the case of an incident solitary wave. The solution describes both the reflected and transmitted waves in the far field, as well as the diffraction in the near field (in the vicinity of the jump). We generalize the solution to the case of a symmetric $n$-layered bar. We show that delamination can lead to the fission of an incident solitary wave, and obtain explicit formulas for the number, amplitudes, velocities, and positions of the secondary solitary waves propagating in each layer of the split waveguide. We establish that generally there is a higher-order reflected wave even when the leading order reflected wave is absent.
\end{abstract}

DOI: 10.1103/PhysRevE.77.066603

PACS number(s): 43.25.+y, 46.40.Cd, 62.30.+d

\section{INTRODUCTION}

The study of nonlinear waves, especially solitons, has been an important theme of recent research in physics. This research included an intensive study of longitudinal bulk solitary waves in nonlinearly elastic waveguides (see [1] and references therein). One of the first long wave models used to describe waves propagating in solid waveguides was the Korteweg-de Vries (KdV) equation [2-4]. The limitations of this model have been discussed in [5]. It has been shown, in particular, that long longitudinal bulk solitary waves in an elastic rod are governed by the so-called doubly dispersive equation (DDE), which is an equation of Boussinesq type with two kinds of dispersive terms [5]. Formal results of the study have been confirmed by successful pioneering experiments on generation and observation of bulk solitary waves in optically transparent polymeric materials such as polystyrene (PS) and plexiglas (PMMA) [6]. Very recently the solitary waves were also observed in the uranium crystalline lattice [7], confirming strong influence of nonlinearity on wave propagation in metals.

Lattice modeling of nonlinear waves in a bilayer with delamination in [8] has shown the sensitivity of a nonlinear wave to certain delamination areas, and invited further detailed studies (see also [9]). Previously, numerical simulations in [1] have shown formation of the second soliton in a rod with an abrupt change of the cross-section radius. Such investigations are of particular interest due to the recent experiments, showing that longitudinal bulk strain solitary

\footnotetext{
*Corresponding author; FAX: +44 (0)1509 223969; k.khusnutdinova@lboro.ac.uk
}

waves can propagate for considerable distances without any significant decay $[10,11]$, inviting investigations into a possibility of using such waves for nondestructive testing (NDT) of certain solid structures.

Various types of layered (or laminated) waveguides are used in physics and engineering to improve deformation properties of elastic structures. Commonly used methods of nondestructive testing of such structures are based on ultrasonic technology and include normal and oblique ultrasonic scans, resonant ultrasonic spectroscopy, and the Lamb wave NDT [12]. These methods detect such inhomogeneities as delamination, voids, porosity, cracks, poor adhesion, etc. However, certain limitations of these methods (e.g., rapid decay of ultrasonic waves in solids [13]), as well as recent theoretical and experimental developments in the area of nonlinear waves stimulate both analytical and experimental research into the approaches. Studies devoted to the propagation and evolution of nonlinear elastic waves in delaminated waveguides are of considerable theoretical and practical interest, in particular, in the view of their possible applications to nondestructive testing of adhesively bonded materials and structures. Such studies are aimed at finding experimentally observable nonlinear physical effects caused by damage or delamination.

Our current research is focused on nonlinear wave processes in layered elastic waveguides with inhomogeneities modeling delamination. In this paper we present a theory of fission of an incident bulk solitary wave in a delaminated, layered elastic bar. We study the problem using asymptotic multiple scales expansions and some results from the integrability theory of the KdV equation by the inverse scattering transform (IST). Our approach allows us to describe explicitly not only the leading order reflected and transmitted waves, but also higher-order diffraction in the vicinity of the 


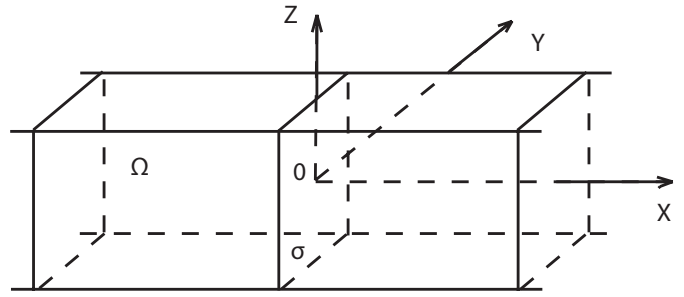

FIG. 1. Elastic bar of rectangular cross section.

"jump" and higher-order corrections to the reflected and transmitted waves.

The phenomenon of fission of a solitary wave is known in theoretical physics of fluids. Indeed, it was shown that when a surface soliton passes through an area of a rapid depth variation, an incident solitary wave evolves into a group of solitons [14-16]. There were also similar studies for internal waves $[17,18]$. The related problem of the evolution of solitons above periodic bottom has been studied in [19], and effects of a randomly rough seabed have been reported in [20]. Soliton dynamics in a strong periodic field has been studied within the KdV framework in [21]. Surprisingly, despite a lot of attention to this phenomenon in various problems of physics of fluids (see above), to the best of our knowledge, fission of a solitary wave has neither been studied theoretically, nor experimentally in any problems of physics of elastic solids.

Our paper is organized as follows. In Sec. II we derive the DDE for long longitudinal waves propagating in a bar of rectangular cross section, in the framework of nonlinear dynamic elasticity. In Sec. III we formulate the problem in terms of the DDE with the "jump" in coefficients, subject to two natural continuity conditions across the "jump." In Sec. IV we consider this problem in a rather general setting and find the weakly nonlinear solution. In Sec. V we study the special case of an incident solitary wave. We show that delamination can lead to the fission of an incident bulk solitary wave and obtain analytical formulas for the number, amplitudes, velocities, and positions of the produced secondary solitary waves. In Sec. VI we consider a generalization of this problem for the case of a symmetric $n$-layered structure. We summarize and discuss our results, and outline some possible extensions of our study in Sec. VII. Some necessary mathematical derivations, and the dimensional form of the solution convenient for the use in physical experiments are given in the appendixes.

\section{MODEL EQUATION FOR LONG NONLINEAR LONGITUDINAL WAVES}

The doubly dispersive equation (DDE) has been derived to describe long nonlinear longitudinal waves in a cylindrical bar of circular cross section in [5]. Here, we derive the DDE equation to describe the propagation of a long nonlinear longitudinal bulk wave in an isotropic elastic bar of rectangular cross section $\sigma=\{-a \leq y \leq a ;-b \leq z \leq b\}$ (Fig. 1). We assume that the bar is in the initial equilibrium state and introduce the Lagrangian cartesian coordinates $(x, y, z)$, where $O x$ is directed along the bar through the center of each crosssection area $\sigma$.

Following the approach developed in [5], we consider the problem in the framework of nonlinear dynamic elasticity, starting from the action functional $S$ written as

$$
S=\int_{t_{0}}^{t_{1}} \int_{\Omega} \mathcal{L}\left(\mathbf{U}, \mathbf{U}_{t}, \mathbf{U}_{x}, \ldots, x, t\right) d \Omega d t,
$$

where $\mathcal{L}\left(\mathbf{U}, \mathbf{U}_{t}, \mathbf{U}_{x}, \ldots, x, t\right)$ is the Lagrangian density per unit volume, $t$ is time, $\Omega$ is a space domain occupied by the waveguide, $\mathbf{U}=\{u, v, w\}$ is the displacement vector in coordinates $(x, y, z)$. The Lagrangian density $\mathcal{L}$ written in material variables is the difference of the kinetic energy density $K$ and the density $\Pi$ of potential energy, as follows:

$$
\mathcal{L}=K-\Pi=\rho(\partial \mathbf{U} / \partial t)^{2} / 2-\rho \Pi\left(I_{k}\right),
$$

where $\rho$ is the density, and $I_{k}=I_{k}(\mathbf{C})$ are the invariants of Cauchy-Green deformation tensor $\mathbf{C}=\left[\nabla \mathbf{U}+(\nabla \mathbf{U})^{T}\right.$ $\left.+\nabla \mathbf{U} \cdot(\nabla \mathbf{U})^{T}\right] / 2$ (e.g., $\left.[22]\right)$,

$$
I_{1}=\operatorname{tr} \mathbf{C}, \quad I_{2}=(1 / 2)\left[(\operatorname{tr} \mathbf{C})^{2}-\left(\operatorname{tr} \mathbf{C}^{2}\right)\right], \quad I_{3}=\operatorname{det} \mathbf{C} .
$$

We shall consider compressible isotropic nonlinearly elastic materials using Murnaghan's model for $\Pi$ [23] and the energy expansion up to the 5-constant approximation as

$$
\Pi=(\lambda+2 \mu) I_{1}^{2} / 2-2 \mu I_{2}+(l+2 m) I_{1}^{3} / 3-2 m I_{1} I_{2}+n I_{3}+\cdots,
$$

where $\lambda$ and $\mu$ are Lame's coefficients, and $l, m, n$ are Murnaghan's moduli.

The complete expressions for the invariants are rather long, can be obtained using any computer algebra package (we used Mathematica [24]), and we shall not give these expressions in the paper. To simplify the problem to the only nonlinear equation for the longitudinal displacement we shall use the planar cross-section hypothesis and the approximate relations for the transverse displacements via the linear longitudinal strain component as follows:

$$
u \approx u(x, t), \quad v \approx-y \nu u_{x}, \quad w \approx-z \nu u_{x},
$$

where $\nu=\lambda /[2(\lambda+\mu)]$ is Poisson's ratio. Similar relations have been introduced in [25] (see also [26,27]) for the case of a cylindrical bar of circular cross section. The description of linear longitudinal waves in a bar of rectangular cross section can be found in $[28,29]$. This description shows that, indeed, relations (3) can be used for the approximate representation of the transverse displacements in a bar of rectangular cross section, under the assumptions made above.

The relations (3) yield the approximate expressions for the invariants valid for the small amplitude long longitudinal elastic waves, and, consequently, to the simplified expressions for $K$ and $\Pi$ per unit volume, as follows:

$$
K=\frac{1}{2} \rho\left(u_{t}^{2}+v_{t}^{2}+w_{t}^{2}\right)=\frac{\rho}{2}\left[u_{t}^{2}+\left(y^{2}+z^{2}\right) \nu^{2} u_{x t}^{2}\right]+\cdots
$$

and 


$$
\Pi=\frac{1}{2}\left(E u_{x}^{2}+\frac{\beta}{3} u_{x}^{3}+\mu \nu^{2}\left(y^{2}+z^{2}\right) u_{x x}^{2}\right)+\cdots,
$$

where the nonlinearity coefficient $\beta$ depends on Murnaghan's moduli $l, m, n$, Young's modulus $E=\mu(3 \lambda+2 \mu)$ / $(\lambda+\mu)$, Poisson's ratio $\nu$, and is given by

$$
\beta=3 E+2 l(1-2 \nu)^{3}+4 m(1+\nu)^{2}(1-2 \nu)+6 n \nu^{2} .
$$

From (1) and (2) we obtain the Euler-Lagrange equation

$$
\begin{aligned}
& \frac{\partial \mathcal{L}_{\sigma}}{\partial u}-\left(\frac{\partial}{\partial t} \frac{\partial \mathcal{L}_{\sigma}}{\partial u_{t}}+\frac{\partial}{\partial x} \frac{\partial \mathcal{L}_{\sigma}}{\partial u_{x}}\right)+\frac{\partial^{2}}{\partial x^{2}} \frac{\partial \mathcal{L}_{\sigma}}{\partial u_{x x}}+\frac{\partial^{2}}{\partial x \partial t} \frac{\partial \mathcal{L}_{\sigma}}{\partial u_{x t}}+\cdots \\
& \quad=0
\end{aligned}
$$

where $\mathcal{L}_{\sigma}=\int_{\sigma} \mathcal{L} d \sigma$ is the Lagrangian density per unit length (see Fig. 1).

Equation (4) yields the DDE for long nonlinear longitudinal displacement waves in a bar of rectangular cross section in a form

$$
u_{t t}-c^{2} u_{x x}=\frac{\beta}{\rho} u_{x} u_{x x}+\frac{J \nu^{2}}{\sigma}\left(u_{t t}-c_{1}^{2} u_{x x}\right)_{x x} .
$$

Here we introduced the linear longitudinal wave velocity $c=\sqrt{E / \rho}$, the linear shear wave velocity $c_{1}=\sqrt{\mu / \rho}$ $=c / \sqrt{2(1+\nu)}$, and the polar moment of inertia of the rectangular cross section $\sigma, J=\int_{\sigma}\left(y^{2}+z^{2}\right) d \sigma=4 a b\left(a^{2}+b^{2}\right) / 3$. Then, $J / \sigma=\left(a^{2}+b^{2}\right) / 3$.

Up to the change of the coefficient of the dispersive terms, (5) is the same as for a cylindrical bar of circular cross section of a radius $R$, when $J=\pi R^{4} / 2$ and $J / \sigma=R^{2} / 2$ [5]. The equation could be derived for a bar of arbitrary cross section, provided that one can justify the validity of relations (3). Note that Eq. (5) is invariant with respect to any shifts of $x, y$, and $z$.

Differentiating (5) with respect to $x$, we obtain the following equation for the linear strain component $e \equiv u_{x}$ :

$$
e_{t t}-c^{2} e_{x x}=\frac{\beta}{2 \rho}\left(e^{2}\right)_{x x}+\frac{J \nu^{2}}{\sigma}\left(e_{t t}-c_{1}^{2} e_{x x}\right)_{x x} .
$$

The one-parameter family of exact solitary wave solutions to (6) can be written in the form

$$
e=e_{0} \operatorname{sech}^{2} \frac{x-s t}{\Lambda}
$$

where

$$
e_{0}=\frac{3 E}{\beta}\left(\frac{s^{2}}{c^{2}}-1\right), \quad \Lambda^{2}=4 \delta \frac{s^{2}-c_{1}^{2}}{s^{2}-c^{2}}, \quad \delta=\frac{J \nu^{2}}{\sigma} .
$$

The amplitude and the width of a solitary wave depend on physical and geometrical properties of a waveguide via four parameters: $c, E / \beta, c_{1}$ and the dispersion parameter $\delta$. When the value of $s$ is close enough to $c$, say, $s=c\left(1+\varepsilon s_{1}+\cdots\right)$, then (7) can be approximately written as

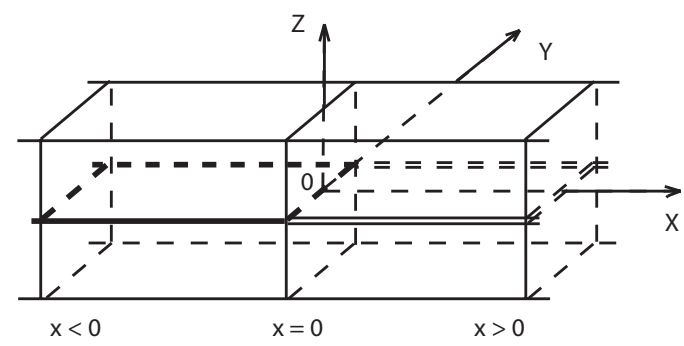

FIG. 2. Delaminated, two-layered elastic bar.

$$
e \approx \varepsilon \tilde{e}=\varepsilon \frac{6 E s_{1}}{\beta} \operatorname{sech}^{2} \sqrt{s_{1}} \frac{\xi-c s_{1} T}{\sqrt{2 \delta\left(1-c_{1}^{2} / c^{2}\right)}},
$$

where $\xi=\sqrt{\varepsilon}(x-c t), T=\varepsilon^{3 / 2} t$, and $\varepsilon s_{1} \approx \frac{s-c}{c}$. The approximation (9) satisfies the $\mathrm{KdV}$ equation

$$
\widetilde{e}_{T}+\frac{1}{2} \frac{\beta c}{E} \widetilde{e} \widetilde{e}_{\xi}+\frac{\delta c}{2}\left(1-\frac{c_{1}^{2}}{c^{2}}\right) \widetilde{e}_{\xi \xi \xi}=0,
$$

derivable from (6) using the asymptotic multiple scales expansion procedure. Note, however, that the $\mathrm{KdV}$ equation (10) should not be used outside the region of its formal validity. The DDE has several advantages as a long wave model, describing, for example, both right-propagating and left-propagating waves. However, the equation is not integrable (see [1]), while the $\mathrm{KdV}$ approximation allows one to solve the initial value problem by the inverse scattering transform.

\section{PROBLEM FORMULATION}

Let us consider the propagation of long nonlinear longitudinal bulk waves in a delaminated, symmetric two-layered elastic bar, as shown in Fig. 2. We assume that there is a perfect interface when $x<0$ and complete debonding (splitting) when $x>0$. The last condition means that for $x>0$ layers are not in contact, and the free surface boundary conditions apply, i.e., all Piola-Kirchhoff stress tensor components should vanish at the free surface of both layers.

Isotropic material to the left-hand side of $x=0$ may be different from (another) isotropic material to the right-hand side, in which case we assume that the cross section $x=0$ is also a perfect interface. Layers are identical and have the same width $2 a$ and the same height $b$.

Assuming that the delamination area is $\{x>0$, $-a<y<a, z=0\}$, using the planar cross-section hypothesis and the symmetry of the structure, we consider the $(1+1)$-dimensional formulation of the problem and use Eq. (5), derived in the preceding section. Namely, we need to find two functions, $u^{-}(x, t)$ for $x<0$ and $u^{+}(x, t)$ for $x>0$, satisfying the two DDEs,

$$
u_{t t}^{ \pm}-c_{ \pm}^{2} u_{x x}^{ \pm}=\delta_{ \pm}\left(u_{t t}^{ \pm}-c_{1 \pm}^{2} u_{x x}^{ \pm}\right)_{x x}+\frac{\beta_{ \pm} c_{ \pm}^{2}}{E_{ \pm}} u_{x}^{ \pm} u_{x x}^{ \pm},
$$

in their respective domains of validity, subject to continuity of longitudinal displacement,

$$
\left.u^{-}\right|_{x=0}=\left.u^{+}\right|_{x=0}
$$

and normal stress, 


$$
\begin{gathered}
c_{-}^{2} u_{x}^{-}+\left.\left(\delta_{-}\left(u_{t t}^{-}-c_{1-}^{2} u_{x x}^{-}\right)_{x}+\frac{1}{2} \frac{\beta_{-} c_{-}^{2}}{E_{-}} u_{x}^{-2}\right)\right|_{x=0} \\
=c_{+}^{2} u_{x}^{+}+\left.\left(\delta_{+}\left(u_{t t}^{+}-c_{1+}^{2} u_{x x}^{+}\right)_{x}+\frac{1}{2} \frac{\beta_{+} c_{+}^{2}}{E_{+}} u_{x}^{+2}\right)\right|_{x=0} .
\end{gathered}
$$

Here parameters of the waveguide to the left-hand side (a thick bar of rectangular cross section $2 a \times 2 b$ ) or to the righthand side (a thin bar of rectangular cross section $2 a \times b$ ) of $x=0$ are denoted by the minus or plus indices, respectively.

To reduce the number of parameters in the problem and to develop the weakly nonlinear theory we define the dimensionless variables as follows:

$$
\tilde{t}=\frac{t}{T^{*}}, \quad \tilde{x}=\frac{x}{X^{*}}, \quad \tilde{u}^{ \pm}=\frac{u^{ \pm}}{U^{*}},
$$

where

$$
T^{*}=\frac{X^{*}}{c_{-}}, \quad U^{*}=-\varepsilon \frac{12 E_{-}}{\beta_{-}} X^{*},
$$

and

$$
X^{*}=\sqrt{\varepsilon^{-1} \frac{\delta_{-}}{2}\left(1-\frac{c_{1-}^{2}}{c_{-}^{2}}\right)} .
$$

The introduction of the small parameter

$$
\varepsilon=\frac{\delta_{-}}{2 X^{* 2}}\left(1-\frac{c_{1-}^{2}}{c_{-}^{2}}\right)=\frac{\delta_{-}}{X^{* 2}} \frac{1+2 \nu_{-}}{4\left(1+\nu_{-}\right)}
$$

is consistent with the assumption that waves under consideration are long $\left(\delta_{-} / X^{* 2} \ll 1\right)$. The linear dispersion is balanced by nonlinearity, yielding the second relation in (11), which is consistent with the assumption of small deformation $\left(U^{*} / X^{*} \ll 1\right)$. The choice of a sign in $U^{*}$ is motivated by a sign of nonlinearity coefficient $\beta_{ \pm}$, which should be negative for the existence of a compression transonic strain solitary wave (see [1]).

Then, up to $O(\varepsilon)$ terms, the problem assumes the following form (tildes are omitted for brevity):

$$
\begin{gathered}
u_{t t}^{-}-u_{x x}^{-}=2 \varepsilon\left(-6 u_{x}^{-} u_{x x}^{-}+u_{x x x x}^{-}\right), \\
u_{t t}^{+}-c^{2} u_{x x}^{+}=2 \varepsilon\left(-6 \beta u_{x}^{+} u_{x x}^{+}+\gamma u_{x x x x}^{+}\right), \\
\left.u^{-}\right|_{x=0}=\left.u^{+}\right|_{x=0}, \\
u_{x}^{-}+\left.2 \varepsilon\left[-3\left(u_{x}^{-}\right)^{2}+u_{x x x}^{-}\right]\right|_{x=0}=c^{2} u_{x}^{+}+2 \varepsilon\left[-3 \beta\left(u_{x}^{+}\right)^{2}\right. \\
\left.+\gamma u_{x x x}^{+}\right]\left.\right|_{x=0} .
\end{gathered}
$$

Here, we used asymptotic relations $u_{t t x x}^{-}=u_{x x x x}^{-}+O(\varepsilon)$ and $u_{t t x x}^{+}=c^{2} u_{x x x x}^{+}+O(\varepsilon)$, and introduced dimensionless parameters

$$
c^{2}=\frac{c_{+}^{2}}{c_{-}^{2}}, \quad \beta=\frac{\beta_{+} c_{+}^{2} / E_{+}}{\beta_{-} c_{-}^{2} / E_{-}}, \quad \gamma=\frac{\delta_{+}\left(c_{+}^{2}-c_{1+}^{2}\right)}{\delta_{-}\left(c_{-}^{2}-c_{1-}^{2}\right)} .
$$

Parameters $c$ and $\beta$ should not be confused with $c$ and $\beta$ introduced in Sec. II, which are no longer used. In the fol- lowing we assume all dimensionless parameters to be of $O(1)$.

\section{WEAKLY NONLINEAR SOLUTION}

We suppose that the incident wave, propagating from the left, is given, and determine both the reflected and the transmitted waves, as well as the diffraction field in the vicinity of the jump. To solve this problem we match two asymptotic multiple scales expansions (for $x<0$ and $x>0$ ) and use some results from the theory of integrable systems.

For $x<0$, we look for a leading order solution in the form of a superposition of an incident wave and a reflected wave, with higher-order terms accounting for the diffraction in the vicinity of the jump and corrections to the reflected wave,

$$
u^{-}=I\left(\xi_{-}, X\right)+R\left(\eta_{-}, X\right)+\varepsilon P\left(\xi_{-}, \eta_{-}, X\right)+O\left(\varepsilon^{2}\right),
$$

where $\xi_{-}=x-t$ and $\eta_{-}=x+t$ are the fast characteristic variables, and $X=\varepsilon x$ is the slow space variable. This ansatz is similar to that introduced in [30] in the study of the interaction of solitons, apart from the replacement of the slow time with the slow space variable.

For $x>0$ we look for a solution in the form of a leading order transmitted wave and higher-order corrections:

$$
u^{+}=T\left(\xi_{+}, X\right)+\varepsilon Q\left(\xi_{+}, \eta_{+}, X\right)+O\left(\varepsilon^{2}\right),
$$

where, again, we introduce the characteristic variables $\xi_{+}=x-c t, \eta_{+}=x+c t$, and the slow space variable $X=\varepsilon x$.

Substituting (17) into (12), we find that the leading order terms cancel, while in $O(\varepsilon)$ we obtain the following equation:

$$
\begin{aligned}
-2 P_{\xi_{-} \eta_{-}}= & \left(I_{X}-3 I_{\xi_{-}}^{2}+I_{\xi_{-} \xi_{-} \xi_{-}}\right)_{\xi_{-}}+\left(R_{X}-3 R_{\eta_{-}}^{2}+R_{\eta_{-} \eta_{-} \eta_{-}}\right)_{\eta_{-}} \\
& -6\left(R I_{\xi_{-}}+I R_{\eta_{-}}\right)_{\xi_{-} \eta_{-}},
\end{aligned}
$$

which yields

$$
\begin{aligned}
-2 P= & \left(I_{X}-3 I_{\xi_{-}}^{2}+I_{\xi_{-} \xi_{-} \xi_{-}}\right) \eta_{-}+\left(R_{X}-3 R_{\eta_{-}}^{2}+R_{\eta_{-} \eta_{-} \eta_{-}}\right) \xi_{-} \\
& -6\left(R I_{\xi_{-}}+I R_{\eta_{-}}\right)-\phi\left(\xi_{-}, X\right)-\psi\left(\eta_{-}, X\right) .
\end{aligned}
$$

We assume that the right-propagating incident wave $I\left(\xi_{-}, X\right)$ is known and defined by a solution of the $\mathrm{KdV}$ equation,

$$
I=\int \tilde{I} d \xi_{-},
$$

where

$$
\widetilde{I}_{X}-6 \widetilde{I I}_{\xi_{-}}+\widetilde{I}_{\xi_{-} \xi_{-} \xi_{-}}=0 .
$$

We need to find the reflected wave

$$
R=\int \tilde{R} d \eta_{-}
$$

where

$$
\tilde{R}_{X}-6 \tilde{R} \tilde{R}_{\eta_{-}}+\widetilde{R}_{\eta_{-} \eta_{-} \eta_{-}}=0,
$$

and the higher-order terms 


$$
P=3\left(R I_{\xi_{-}}+I R_{\eta_{-}}\right)+\phi\left(\xi_{-}, X\right)+\psi\left(\eta_{-}, X\right) .
$$

We choose the function $\phi\left(\xi_{-}, X\right)=0$ in accordance with the usual radiation conditions (there must be no corrections to the given incident wave in the disturbance caused by it), and the function $\psi\left(\eta_{-}, X\right)$ must be found from the continuity conditions (14) and (15).

Similarly, in the delaminated area $(x>0)$ we obtain

$$
-2 c^{2} Q_{\xi_{+} \eta_{+}}=\left(c^{2} T_{X}-3 \beta T_{\xi_{+}}^{2}+\gamma T_{\xi_{+} \xi_{+} \xi_{+}}\right)_{\xi_{+}}
$$

with $\beta$ and $\gamma$ from (16).

Therefore, we look for the leading order transmitted wave

$$
T=\int \widetilde{T} d \xi_{+},
$$

where

$$
\widetilde{T}_{X}-6 \frac{\beta}{c^{2}} \widetilde{T} \widetilde{T}_{\xi_{+}}+\frac{\gamma}{c^{2}} \widetilde{T}_{\xi_{+} \xi_{+} \xi_{+}}=0
$$

and the higher-order corrections

$$
Q=q\left(\xi_{+}, X\right)+r\left(\eta_{+}, X\right) .
$$

Here again $r\left(\eta_{+}, X\right)=0$ due to the usual radiation conditions (if the incident wave is coming only from the left, the waves on the right-hand side must be right going), and $q\left(\xi_{+}, X\right)$ should be found from the continuity conditions (14) and (15).

Now we shall use (14) and (15) to find the "initial" data for two KdV equations (22) and (23), and define the functions $\psi\left(\eta_{-}, X\right)$ and $q\left(\xi_{+}, X\right)$. Although $X=0$ if $x=0$, in what follows we shall formally trace the inherited dependence of $\psi\left(\eta_{-}, X\right)$ and $q\left(\xi_{+}, X\right)$ on $X$.

Condition (14) implies

$$
\left.u_{t}^{-}\right|_{x=0}=\left.u_{t}^{+}\right|_{x=0} .
$$

At leading order, this yields

$$
\left.I_{\xi_{-}}\right|_{x=0}-\left.R_{\eta_{-}}\right|_{x=0}=\left.c T_{\xi_{+}}\right|_{x=0},
$$

while $O(\varepsilon)$ terms give

$$
\left.\psi_{\eta_{-}}\right|_{x=0}+\left.c q_{\xi_{+}}\right|_{x=0}=\left.3\left(R I_{\xi_{-} \xi_{-}}-I R_{\eta_{-} \eta_{-}}\right)\right|_{x=0}=\left.f(t, X)\right|_{x=0} .
$$

The right-hand side here is the function of $t$ and $X$, denoted by $f(t, X)$. Similarly, condition (15) implies

$$
\left.I_{\xi_{-}}\right|_{x=0}+\left.R_{\eta_{-}}\right|_{x=0}=\left.c^{2} T_{\xi_{+}}\right|_{x=0}
$$

and

$$
\begin{aligned}
\left.\psi_{\eta_{-}}\right|_{x=0}-\left.c^{2} q_{\xi_{+}}\right|_{x=0}= & {\left[-\left(I_{X}-6 I_{\xi_{-}}^{2}+2 I_{\xi_{-} \xi_{-} \xi_{-}}\right)-\left(R_{X}-6 R_{\eta_{-}}^{2}\right.\right.} \\
& \left.+2 R_{\eta_{-} \eta_{-} \eta_{-}}\right)+c^{2} T_{X}-6 \beta T_{\xi_{+}}^{2}+2 \gamma T_{\xi_{+} \xi_{+} \xi_{+}} \\
& \left.-3\left(R I_{\xi_{-} \xi_{-}}+I R_{\eta_{-} \eta_{-}}\right)\right]_{x=0}=\left.g(t, X)\right|_{x=0},
\end{aligned}
$$

where, again, the right-hand side is the function of $t$ and $X$, denoted by $g(t, X)$.
Then, from (24) and (26) we can find the "initial" conditions for the KdV equations (22) and (23), defining both reflected and transmitted "strain" waves at $x=0$ in terms of the given incident wave

$$
\left.\widetilde{R}\right|_{x=0}=\left.C_{R} \widetilde{I}\right|_{x=0} \quad \text { and }\left.\quad \widetilde{T}\right|_{x=0}=\left.C_{T} \widetilde{I}\right|_{x=0},
$$

where we introduced the reflection coefficient

$$
C_{R}=\frac{c-1}{c+1},
$$

and the transmission coefficient

$$
C_{T}=\frac{2}{c(1+c)} .
$$

Note that if materials in the bonded and delaminated areas are identical, then $c_{-}=c_{+}$, yielding $c=1$ and $C_{R}=0$, and there will be no leading order reflected wave.

We can now simplify (25) and (27) using (21)-(23) and (28) to obtain

$$
\begin{aligned}
&\left.f(t, X)\right|_{x=0}=\left[3\left(R+C_{R} I\right) I_{\xi_{-} \xi_{-}}\right]_{x=0}, \\
&\left.g(t, X)\right|_{x=0}=\left\{3\left[\left(1+C_{R}\right)^{2}-\beta C_{T}^{2}\right] I_{\xi_{-}}^{2}-\left(1+C_{R}\right.\right. \\
&\left.\left.-\gamma c^{-2} C_{T}\right) I_{\xi_{-} \xi_{-} \xi_{-}}-3\left(R-C_{R} I\right) I_{\xi_{-} \xi_{-}}\right\}_{x=0} .
\end{aligned}
$$

From (25) and (27) we find

$$
\begin{aligned}
\left.\psi_{\eta_{-}}\right|_{x=0} & =\left(\frac{1}{1+c}[c f(t, X)+g(t, X)]\right)_{x=0}, \\
\left.q_{\xi_{+}}\right|_{x=0} & =\left(\frac{1}{c(1+c)}[f(t, X)-g(t, X)]\right)_{x=0},
\end{aligned}
$$

where by now both functions $f(t, X)$ and $g(t, X)$ are completely defined in terms of the leading order incident, reflected and transmitted waves. These conditions allow us to restore the dependence of both functions on their respective characteristic variables

$$
\begin{gathered}
\psi\left(\eta_{-}, X\right)=\frac{1}{1+c} \int\left[c f\left(\eta_{-}, X\right)+g\left(\eta_{-}, X\right)\right] d \eta_{-}, \\
q\left(\xi_{+}, X\right)=\frac{1}{c(1+c)} \int\left[f\left(-\frac{\xi_{+}}{c}, X\right)-g\left(-\frac{\xi_{+}}{c}, X\right)\right] d \xi_{+},
\end{gathered}
$$

while, with the same accuracy as in the formulation of the problem, the dependence of both functions on the slow space variable $X$ is inherited from the dependence of the leading order incident, reflected and transmitted waves.

Note that constants of integration in (21)-(23) and (33) should be found from some additional physical conditions. In the next section we require that both the incident and transmitted waves propagate into unperturbed medium, and use the first continuity condition (14). 


\section{FISSION OF AN INCIDENT STRAIN SOLITARY WAVE}

Although the weakly nonlinear solution obtained in the preceding section can be applied to various types of an incident wave [given by a solution of the $\mathrm{KdV}$ equation (21)], here we consider a special case of an incident solitary wave. This problem is particularly important from the viewpoint of our current experimental research of nonlinear waves in delaminated, polymeric layered waveguides [31].

We assume that the leading order right-propagating incident "strain" wave is given by an exact solitary wave solution of (21),

$$
\tilde{I}=-\frac{v}{2} \operatorname{sech}^{2} \frac{\sqrt{v}}{2}\left(\xi_{-}-v X\right) .
$$

The incident wave propagates into the unperturbed medium,

$$
\lim _{t \rightarrow-\infty}\left(\left.I\right|_{x=0}\right)=0 \text {, }
$$

which leads, by integration of $\tilde{I}$, to

$$
I=-\sqrt{v}\left(\tanh \frac{\sqrt{v}}{2}\left(\xi_{-}-v X\right)-1\right) .
$$

We should describe the leading order reflected and transmitted waves, as well as the higher-order corrections. Since experimentally measured quantities are the "strains" $u_{x}^{-}$and $u_{x}^{+}$ (see, for example, [1]), we aim at finding the explicit leading order asymptotics of these functions for large $t$ and $x$.

\section{A. Transmitted wave}

The leading order transmitted wave field in the delaminated area can be described using the results from the integrability theory of the $\mathrm{KdV}$ equation (see, e.g., [32,33]) and our formulas (23), (28), and (30) for the transmitted wave.

Let us write (23) in the canonical form

$$
U_{\tau}-6 U U_{\chi}+U_{\chi \chi \chi}=0
$$

by introduction of the variables

$$
U=\frac{\beta}{\gamma} \tilde{T}, \quad \tau=\frac{\gamma}{c^{2}} X, \quad \chi=\xi_{+} .
$$

Then, the transmitted wave field is defined by the spectrum of the corresponding Schrödinger equation

$$
\Psi_{\chi \chi}+[\lambda-U(\chi)] \Psi=0,
$$

where, in accordance with (28) and (35), the potential is given by

$$
U(\chi)=-A \operatorname{sech}^{2} \frac{\chi}{l}, \quad A=\frac{v \beta}{\gamma c(1+c)}, \quad l=\frac{2 c}{\sqrt{v}} .
$$

The sign of $A$ depends on the sign of the ratio $\beta_{+} / \beta_{-}$. If $A<0$, then the transmitted wave field will not contain any solitary waves. Instead, the "initial" pulse will degenerate into a dispersive wave train (see [32]). If, however, the coefficient $A$ is positive (e.g., when the whole structure is made of materials, supporting strain solitons of one and the same type), there always will be at least one discrete eigenvalue, i.e., at least one solitary wave in the transmitted wave field, accompanied by the radiation corresponding to the continuous spectrum. However, in some cases more than one secondary soliton can be produced from a single incident solitary wave resulting in the so-called fission of a soliton [14-16].

Note that (37) is not an exact stationary solitary wave solution of (23). The discrete eigenvalues of (36) for such a potential are given in [34] (see also $[32,33]$ ) by

$$
\lambda=-k_{n}^{2} \text {, }
$$

where

$$
k_{n}=\frac{1}{2 l}\left[\left(1+4 A l^{2}\right)^{1 / 2}-(2 n-1)\right]>0, \quad n=1,2, \ldots, N .
$$

The number $N$ of solitary waves produced in the delaminated area is defined by the largest integer, satisfying the inequality

$$
N<\frac{1}{2}\left[\left(1+\frac{4 \alpha^{2}}{\pi^{2}}\right)^{1 / 2}+1\right],
$$

where

$$
\alpha=\pi \sqrt{A} l=2 \pi \sqrt{\frac{\beta}{\gamma} \frac{c}{1+c}} .
$$

Parameters $\beta, \gamma$, and $c$ depend on material properties and geometry of the waveguide, and have been defined in Sec. III. There is always one solitary wave for small $\alpha$, while more solitons will emerge as $\alpha$ increases. Asymptotically, as $\tau \rightarrow+\infty$, the solution evolves into a train of solitary waves propagating to the right, and some dispersive radiation (a dispersive wave train) propagating to the left (see [32]):

$$
U \sim-\sum_{n=1}^{N} 2 k_{n}^{2} \operatorname{sech}^{2} k_{n}\left(\chi-4 k_{n}^{2} \tau-\chi_{n}\right)+\text { radiation, }
$$

where the phase shifts $\chi_{n}$ are given in Appendix A.

Is the soliton fission still possible if the waveguide is made of one and the same material?

The answer is entirely defined by the geometry of the waveguide. Indeed, in this case, $c=1, \beta=1$, and $\gamma=\left(J_{+} \sigma_{-}\right) /\left(J_{-} \sigma_{+}\right)=\left(4+\kappa^{2}\right) /\left[4\left(1+\kappa^{2}\right)\right]$, where $\kappa=b / a$, since $J_{-} / \sigma_{-}=\left(a^{2}+b^{2}\right) / 3$ and $J_{+} / \sigma_{+}=\left(a^{2}+b^{2} / 4\right) / 3$. This yields

$$
\frac{\alpha}{\pi}=\sqrt{\frac{2}{\gamma}}
$$

where

$$
\gamma=\frac{4+\kappa^{2}}{4\left(1+\kappa^{2}\right)}
$$

Then,

$$
N=\left[\text { largest integer }<\frac{1}{2}\left(\sqrt{1+32 \frac{1+\kappa^{2}}{4+\kappa^{2}}}+1\right)\right] .
$$

The number of secondary solitons depends on $\kappa$, and there will be either two (for $\kappa \leqslant 2 \sqrt{2}$ ) or three (for $\kappa>2 \sqrt{2}$ ) soli- 


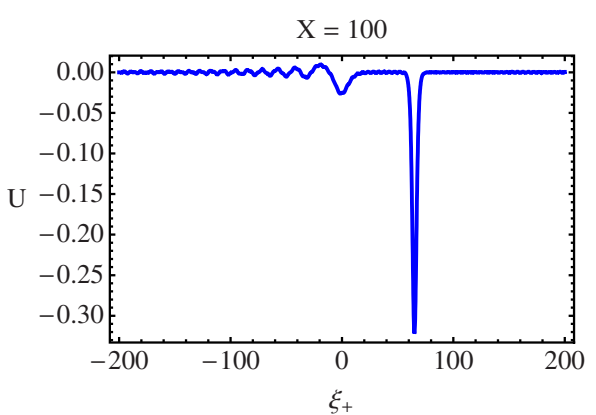

FIG. 3. (Color online) Two secondary solitons and decaying radiation in the transmitted wave field at $X=100(\kappa=1, v=0.35)$.

tons. For example, for $\kappa=1$ (i.e., for square cross section $b=a$ ) there will be two secondary solitons in the delaminated area. Numerical solution with Mathematica [24] of the $\mathrm{KdV}$ Eq. (23) for $\kappa=1, v=0.35$ is shown in Fig. 3 for $X=100$. The three-dimensional image of $-U$ is shown in Fig. 4.

The results of this section invite a detailed experimental verification of the fission of a strain solitary wave in a delaminated elastic bar. In particular, for a waveguide made of one and the same material our theory yields the following theoretical prediction for the ratio $C_{T}^{A_{1}}$ of the amplitude of the lead (first) "strain" solitary wave in the delaminated area to the amplitude of the incident "strain" solitary wave in the bonded area:

$$
C_{T}^{A_{1}}=\frac{\gamma}{4}\left(\sqrt{1+\frac{8}{\gamma}}-1\right)^{2} .
$$

The dimensional expressions for the incident and transmitted solitons are given in Appendix B.

Some preliminary results of the observation of the soliton fission in solids may be found in [31]. An initial solitary density (or bulk) wave was generated, using "the bulk soliton factory," i.e., an experimental setup described in detail in $[6,1]$. In these first experiments an incident bulk solitary wave propagates in a $10 \times 10 \times 400 \mathrm{~mm}$ PMMA bar, divided

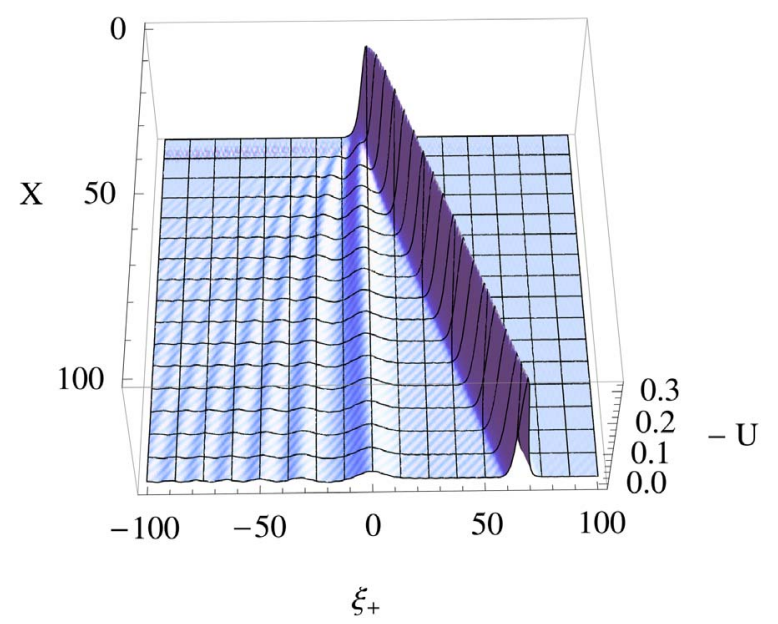

FIG. 4. (Color online) The emergence of two secondary solitons and decaying radiation in the transmitted wave field $(\kappa=1$, $v=0.35)$. in two symmetric parallel parts by a thin longitudinal cut. The cut starts at $70 \mathrm{~mm}$ from the input cross section of the bar, and is parallel to the recording laser light. Our subsequent first experiments with delaminated, layered polymeric bars (for a type of a glue, when an interface can be considered to be "perfect") show the following: a longitudinal bulk strain solitary wave, generated in a glued part, fissions, and considerably changes due to delamination, similar to experiments with the split bar. The amplitude of the lead (first) soliton increases, its width decreases in the split area in accordance with the theoretical predictions. In particular, the increase of the soliton amplitude is experimentally detectable and lies in the interval of 10\%-20\% (see [31]). The experimentally measured amplification of a soliton is in rather good agreement with that predicted by our theory. Details of the expanded and refined experimental studies of soliton fission in solids will be presented elsewhere.

\section{B. Reflected wave}

To describe the leading order reflected wave field we use formula (22), already written in the canonical form, as well as (28) and (29). Therefore, the wave field is defined by the spectrum of the Schrödinger equation (36), where, in accordance with (28), the potential $U(\chi)$ is given by

$$
U(\chi)=-B \operatorname{sech}^{2} \frac{\chi}{m}, \quad B=\frac{v(c-1)}{2(c+1)}, \quad m=\frac{2}{\sqrt{v}} .
$$

The sign of $B$ depends on the sign of the reflection coefficient $C_{R}=(c-1) /(c+1)$, and is negative, if $c<1$. In this case the reflected wave field does not contain any solitary waves, and the "initial" pulse degenerates into a dispersive wave train. If $c>1$, there will be at least one reflected solitary wave accompanied by radiation. To describe the solitary waves, one can use formulas (38) and (39), changing $A \rightarrow B$ and $l \rightarrow m$.

If the structure is made of one and the same material, then the reflection coefficient $C_{R}=0$, and there will be no leading order reflected wave.

\section{Higher-order corrections}

To find the higher-order corrections to the wave fields we need to calculate the functions $f(t, X)$ and $g(t, X)$, given by (31). Details of these calculations are shown in Appendix C. As mentioned above, for $c=1$ there is no leading order reflected wave. However, the analysis of higher-order corrections to the "strain" field $u_{x}^{-}$shows that there is a small (i.e., higher-order) reflected wave even for $c=1$ (see Appendix C).

\section{FISSION IN A DELAMINATED, SYMMETRIC MULTILAYERED BAR}

The results obtained in preceding sections can be generalized for the problem of the long nonlinear longitudinal bulk waves in a symmetric $n$-layered elastic bar, under the assumption that all interfaces are perfect when $x<0$ and completely debonded when $x>0$. We assume that layers are identical, of the width $2 a$ and the height $2 b / n$. Again, the 
TABLE I. The number $N$ of secondary solitary waves for some values of $\kappa$ and $n$.

\begin{tabular}{lccccccccc}
\hline \hline & & $n=2$ & \multicolumn{1}{c}{$n=3$} & \multicolumn{3}{c}{$4 \leq n \leq 8$} \\
\hline$\kappa=b / a$ & 1 & 2 & 3 & 1 & 2 & 3 & 1 & 2 & 3 \\
$N$ & 2 & 2 & 3 & 2 & 3 & 3 & 2 & 3 & 4 \\
\hline \hline
\end{tabular}

material on the left-hand side of the $x=0$ cross section can be different from the material on the right-hand side, in which case we assume that $x=0$ is a perfect interface. In the same dimensionless variables as before, the one-dimensional formulation of the problem assumes the form (12)-(15). The only difference will be in the numerical value of the dimensionless coefficient $\gamma$. Indeed, if, for example, all elements of the structure are made of one and the same material, we have

$$
\gamma=\gamma(n, \kappa)=\frac{n^{2}+\kappa^{2}}{n^{2}\left(1+\kappa^{2}\right)}, \quad \text { where } \kappa=b / a,
$$

since now $J_{+} / \sigma_{+}=\left(a^{2}+b^{2} / n^{2}\right) / 3$. Then the number of secondary solitons in the delaminated area is given by

$$
N=\left[\text { largest integer }<\frac{1}{2}\left(\sqrt{1+8 n^{2} \frac{1+\kappa^{2}}{n^{2}+\kappa^{2}}}+1\right)\right] .
$$

It depends on the geometry of the waveguide via the ratio $\kappa=b / a$ and the number of layers $n$. The number $N$ of secondary solitary waves for some values of $\kappa$ and $n$ is shown in Table I.

These results also suggest possible experimental verification of the developed theory by increasing the number $n$ of layers for one and the same value of $\kappa$. In particular, the theoretical prediction for the ratio $C_{T}^{A_{1}}$ of the amplitude of the lead (first) "strain" solitary wave in the delaminated area to the amplitude of the incident "strain" solitary wave in the bonded area is given by (41), where the constant $\gamma$ for the given values of $n$ (the number of layers) and $\kappa$ (the ratio of the full height of the multilayer to its full width) is defined by (42). The general dimensional form of the leading order incident, transmitted and reflected "strain" wave fields is given in Appendix B.

\section{CONCLUDING REMARKS}

We have studied the propagation of a nonlinear longitudinal bulk strain wave in a symmetric delaminated bar. We considered a two-layered bar with the perfect interface when $x<0$, and complete debonding when $x>0$ (the coordinate axis $O x$ is directed along the bar). Matching two asymptotic multiple scales expansions (for $x<0$ and $x>0$ ), we found the weakly nonlinear solution to the corresponding mathematical problem, formulated in terms of the DDE (a Boussinesq-type equation) with the "jump" in coefficients, subject to two natural continuity conditions at the "jump." Using integrability of the $\mathrm{KdV}$ equation, we described fission of an incident solitary wave in a delaminated waveguide. We have predicted the number and described asymptotics of the generated secondary solitary waves, inviting the detailed experimental studies of this phenomenon.
The effect of fission of a strain soliton may prove useful for nondestructive testing and evaluation of various nonlinearly elastic layered structures. Indeed, having generated an input solitary wave at each end of a bonded structure, one can then register the output solitons at the other end, and compare their parameters with the parameters of the incident wave. Clearly, many more experimental and theoretical studies are needed.

In our theory we have simplified the physical problem, using the planar cross section hypothesis and approximate relations for the transverse displacements (3). Obviously, such simplified relations (3) may not provide vanishing stresses on the free lateral surface of a bar with the desired accuracy (see, for example, [29] for the relevant discussion in the context of linear waves). As it was shown in [35] for nonlinear waves in an elastic rod, these relations may be prolonged to obtain a refined version of the nonlinear model equation. For weakly nonlinear waves this refinement results in a small correction to the coefficient of the dispersive term in the Boussinesq equation, derivable from the DDE equation (see Sec. II). An extension of our study will involve the refinement of the DDE (5) with the subsequent corrections to our theory by considering the higher-order corrections to (3), similar to the studies mentioned above.

For waves of moderate amplitude it might be appropriate to extend the DDE with a higher-order cubic nonlinearity [36], similar to a well-known approach in fluid mechanics $[37,38]$. However, these corrections were not considered here because of insufficient and/or unreliable data on the fourthorder elastic moduli of materials. The effect of such higherorder corrections on the fission phenomenon in the context of the Gardner equation has been considered recently in [18].

An interesting generalization of the developed theory can be expected for various problems of multiple delamination areas, both for perfect and imperfect interfaces, for other types of incident waves, and for the more complicated cases of asymmetric layered structures (when either geometrical or physical properties of layers are not identical). We will address these questions in our future studies.

Another application of the considered problem is related to the nonlinear Fermi-Pasta-Ulam (FPU) chains [39], which are currently a subject of active research (see, for example, [40] and references therein). Indeed, the same problem (12)-(15) appears in a continuum approximation for long weakly nonlinear waves in an inhomogeneous FPU chain consisting of two connected parts with different properties. The derivation of a Boussinesq equation for a chain can be found, for example, in [41]. We also note, that the methodology, developed in our paper, can be applied to other problems described by equations with the "jump" in coefficients.

Finally, we would like to mention that the results of our study suggest a realistic experimental setup to observe the phenomenon of fission of a solitary wave in condensed matter physics. The comparison of theoretical predictions for the amplitude variation of the transmitted lead solitary wave with first experimental results for a delaminated two-layered PMMA bar looks encouraging (see [31]), and the detailed experimental study is now in progress. The results will be reported elsewhere. 


\section{ACKNOWLEDGMENTS}

We thank W.A. Green and E.N. Pelinovsky for useful discussions. The research is supported by EPSRC-GB Grant No. EP/D035570/1.

\section{APPENDIX A}

The phase shifts of the solitary waves in (40) are given by

$$
\chi_{1}=\frac{1}{2 k_{1}} \ln \left(\frac{c_{1}}{2 k_{1}}\right)
$$

and

$$
\chi_{n}=\frac{1}{2 k_{n}} \ln \left[\frac{c_{n}}{2 k_{n}} \prod_{m=1}^{n-1}\left(\frac{k_{n}-k_{m}}{k_{n}+k_{m}}\right)^{2}\right] \text { for } n>1 .
$$

The constants $c_{n}$ are equal to

$$
c_{n}=\left(\int_{-\infty}^{\infty} \Psi_{n}^{2}(x) d x\right)^{-1}
$$

where $\Psi_{n}(x)$ is the eigenfunction of (36) corresponding to the $n$th eigenvalue $-k_{n}^{2}$,

$$
\begin{aligned}
\Psi_{n}= & \operatorname{const}\left(1-\tanh ^{2} \frac{x}{l}\right)^{k_{n} l / 2} \\
& \times_{2} F_{1}\left(1-n, 2 k_{n} l+n, k_{n} l+1, \frac{1-\tanh \frac{x}{l}}{2}\right)
\end{aligned}
$$

(see [34]). Here, ${ }_{2} F_{1}(\cdots)$ is the hypergeometric function, and an arbitrary constant should be chosen to normalize the eigenfunction at infinity,

$$
\Psi_{n} \approx e^{-k_{n} x} \quad \text { as } \quad x \rightarrow+\infty .
$$

\section{APPENDIX B}

Here we shall give the expressions for waves in terms of physical parameters, suitable for experiments. Let $\widetilde{I}\left(\xi_{-}, X\right)$, $\widetilde{R}\left(\eta_{-}, X\right)$, and $\widetilde{T}\left(\xi_{+}, X\right)$ be dimensionless leading order incident, reflected and transmitted "strain" waves, respectively. To return to the dimensional physical variables one should use the following formulas:

$$
\begin{aligned}
& u_{x I}^{-}=\frac{U^{*}}{X^{*}} \tilde{I}\left(\frac{x-c_{-} t}{X^{*}}, \frac{\varepsilon}{X^{*}} x\right)+\cdots \\
& u_{x R}^{-}=\frac{U^{*}}{X^{*}} \widetilde{R}\left(\frac{x+c_{-} t}{X^{*}}, \frac{\varepsilon}{X^{*}} x\right)+\cdots \\
& u_{x T}^{+}=\frac{U^{*}}{X^{*}} \widetilde{T}\left(\frac{x-c_{+} t}{X^{*}}, \frac{\varepsilon}{X^{*}} x\right)+\cdots,
\end{aligned}
$$

where $X^{*}$ and $U^{*}$ are given by (11).

We introduce the following auxiliary parameters:

$$
\beta_{1}=\frac{\beta_{-}}{6 E_{-}}, \quad \mu_{1}=1-\frac{\mu_{-}}{E_{-}},
$$

The dimensional form of the incident solitary wave can be given in terms of the strain amplitude as follows:

$$
u_{x I}^{-}=A_{s} \operatorname{sech}^{2} \frac{x-s_{-} t}{\Lambda_{-}},
$$

where

$$
s_{-}=\frac{c_{-}}{1-A_{s} \beta_{1}}, \quad \Lambda_{-}^{2}=\frac{2 \delta_{-} \mu_{1}}{A_{s} \beta_{1}\left(1-A_{s} \beta_{1}\right)^{2}} ;
$$

or in terms of the soliton velocity,

$$
u_{x I}^{-}=\frac{1}{\beta_{1}}\left(1-\frac{c_{-}}{s_{-}}\right) \operatorname{sech}^{2} \frac{c_{-}}{s_{-}}\left(1-\frac{c_{-}}{s_{-}}\right)^{1 / 2} \frac{x-s_{-} t}{\sqrt{2 \delta_{-} \mu_{1}}} .
$$

The transmitted wave field can be written in dimensional form as follows:

$$
\begin{aligned}
u_{x T}^{+}= & 4 \frac{\gamma}{\beta} A_{s} \sum_{n=1}^{N} \widetilde{k}_{n}^{2} \operatorname{sech}^{2} \widetilde{k}_{n}\left\{\sqrt{\frac{2 A_{s} \beta_{1}}{\delta_{-} \mu_{1}}}\right. \\
& \left.\times\left[\left(1-4 A_{s} \beta_{1} \frac{\gamma}{c^{2}} \widetilde{k}_{n}^{2}\right) x-c_{+} t\right]-\widetilde{\chi}_{n}\right\} \\
& + \text { dispersive radiation, }
\end{aligned}
$$

where $\tilde{k}_{n}=k_{n} / \sqrt{v}$ and $\tilde{\chi}_{n}=\sqrt{v} \chi_{n}$, while $\chi_{n}$ is given in (A1).

\section{APPENDIX C}

To find the higher-order corrections to the wave fields we calculate the functions $f(t, X)$ and $g(t, X)$, given by (31), and therefore, the values of functions $I, R$, and $T$ at $x=0$ (as functions of $t$ and $X$ ). The incident wave $I$ is given in (34). Functions $R(t, X)$ and $T(t, X)$ can be found from (28) up to a constant by an appropriate integration with respect to $t$,

$$
\left.R\right|_{x=0}=\int\left(\left.\widetilde{R}\right|_{x=0}\right) d t \quad \text { and }\left.\quad T\right|_{x=0}=-c \int\left(\left.\widetilde{T}\right|_{x=0}\right) d t .
$$

The constant of integration in $T$ can be found from the condition that the transmitted wave propagates into unperturbed medium,

$$
\lim _{t \rightarrow-\infty}\left(\left.T\right|_{x=0}\right)=0
$$

which yields

$$
\left.T\right|_{x=0}=c C_{T} \sqrt{v}\left(\tanh \frac{\sqrt{v}}{2}(t+v X)+1\right) .
$$

The constant of integration in $R$ can be found using the condition

$$
\left.I\right|_{x=0}+\left.R\right|_{x=0}=\left.T\right|_{x=0}
$$

or, equivalently,

$$
\lim _{t \rightarrow-\infty}\left(\left.R\right|_{x=0}\right)=0,
$$

following to leading order from (14). This gives 


$$
\left.R\right|_{x=0}=-C_{R} \sqrt{v}\left(\tanh \frac{\sqrt{v}}{2}(t+v X)+1\right) .
$$

The calculation of $f(t, X)$ and $g(t, X)$ yields

$$
f(t, X)=0
$$

and

$$
\begin{aligned}
g(t, X)= & a \operatorname{sech}^{4} \frac{\sqrt{v}}{2}(t+v X)\left[b_{1}+b_{2} \cosh \sqrt{v}(t+v X)\right. \\
& \left.+b_{3} \sinh \sqrt{v}(t+v X)\right],
\end{aligned}
$$

where

$$
\begin{gathered}
a=\frac{v^{2}}{4}, \\
b_{1}=1+10 C_{R}+3 C_{R}^{2}+2 \gamma c^{-2} C_{T}-3 \beta C_{T}^{2}, \\
b_{2}=1-5 C_{R}-\gamma c^{-2} C_{T}, \\
b_{3}=-6 C_{R} .
\end{gathered}
$$

Consequently, the expansions of the "strain" fields $u_{x}^{-}$and $u_{x}^{+}$ are given by

$$
\begin{aligned}
u_{x}^{-}= & I_{x}\left(\xi_{-}, X\right)+R_{x}\left(\eta_{-}, X\right)+\varepsilon\left(3\left(R \widetilde{I}_{\xi_{-}}+2 \widetilde{I R}+I \widetilde{R}_{\eta_{-}}\right)\right. \\
& \left.+\frac{1}{1+c} g\left(\eta_{-}, X\right)\right)
\end{aligned}
$$

and

$$
u_{x}^{+}=T_{x}\left(\xi_{+}, X\right)-\varepsilon \frac{1}{c(1+c)} g\left(-\frac{\xi_{+}}{c}, X\right),
$$

respectively. Here the corrections in (C5) describe diffraction in the vicinity of the "jump," and higher-order correction to the reflected wave, while (C6) gives higher-order correction to the transmitted wave.

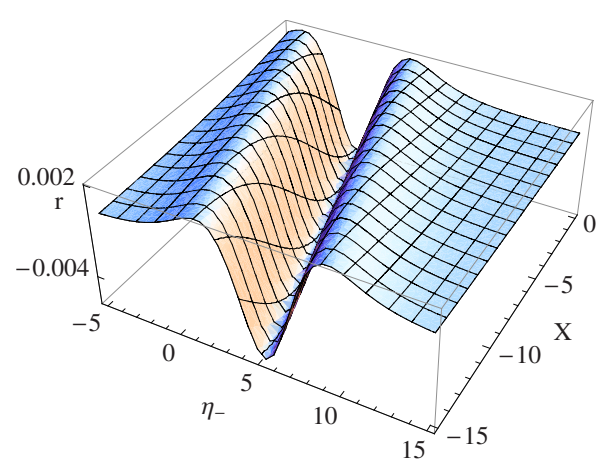

FIG. 5. (Color online) Higher-order reflected wave $r\left(\eta_{-}, X\right)$ for $c=1, \quad \beta=1, \gamma=5 / 8, v=0.35$.

As it was mentioned in Sec. V B, there is no leading order reflected wave if $c=1$. However, as follows from (C5), a small reflected wave exists in higher-order corrections to the "strain" field $u_{x}^{-}$,

$$
u_{x}^{-}=I_{x}\left(\xi_{-}, X\right)+\varepsilon r\left(\eta_{-}, X\right),
$$

where

$$
\begin{aligned}
r\left(\eta_{-}, X\right)= & \frac{v^{2}}{8} \operatorname{sech}^{4} \frac{\sqrt{v}}{2}\left(\eta_{-}+v X\right)[1+2 \gamma-3 \beta \\
& \left.+(1-\gamma) \cosh \sqrt{v}\left(\eta_{-}+v X\right)\right] .
\end{aligned}
$$

The function $r\left(\eta_{-}, X\right)$ is plotted in Fig. 5 for $c=1$, $\beta=1, \quad \gamma=5 / 8(\kappa=1)$ and $v=0.35$.

A similar correction to the transmitted "strain" wave field follows from (C6) for $c=1$ :

$$
\begin{aligned}
u_{x}^{+}= & T_{x}\left(\xi_{+}, X\right)-\varepsilon \frac{v^{2}}{8} \operatorname{sech}^{4} \frac{\sqrt{v}}{2}\left(\xi_{+}-v X\right)[1+2 \gamma-3 \beta \\
& \left.+(1-\gamma) \cosh \sqrt{v}\left(\xi_{+}-v X\right)\right] .
\end{aligned}
$$

Thus, we have explicitly calculated the higher-order corrections to the "strain" fields. We have shown that they do not contain any secular terms, and we have found corrections to both the transmitted and the reflected wave fields.
[1] A. M. Samsonov, Strain Solitons in Solids and How to Construct Them (Chapman and Hall/CRC, Boca Raton, FL, 2001).

[2] G. A. Nariboli and A. Sedov, J. Math. Anal. Appl. 32, 661 (1970).

[3] L. A. Ostrovskii and A. M. Sutin, Appl. Math. Mech. 41, 543 (1977).

[4] J. Engelbrecht, Nonlinear Wave Processes of Deformation in Solids (Pitman, London, 1981).

[5] A. M. Samsonov, Sov. Phys. Dokl. 29, 586 (1984).

[6] G. V. Dreiden, Y. I. Ostrovsky, A. M. Samsonov, I. V. Semenova, and E. V. Sokurinskaya, Sov. Phys. Tech. Phys. 33, 1237 (1988).

[7] M. E. Manley, M. Yethiraj, H. Sinn, H. M. Volz, A. Alatas, J. C. Lashley, W. L. Hults, G. H. Lander, and J. L. Smith, Phys.
Rev. Lett. 96, 125501 (2006).

[8] K. R. Khusnutdinova and V. V. Silberschmidt, Proc. Est. Acad. Sci., Phys., Math. 51, 63 (2003).

[9] K. R. Khusnutdinova, Eur. Phys. J. Spec. Top. 147, 45 (2007).

[10] I. V. Semenova, G. V. Dreiden, and A. M. Samsonov, Proc. SPIE 5880, 32 (2005).

[11] A. M. Samsonov, G. V. Dreiden, and I. V. Semenova, Innovations in Nonlinear Acoustics (AIP, Melville, NY, 2006), Vol. CP-838, p. 147.

[12] E. Maeva, I. Severina, S. Bondarenko, G. Chapman, B. O’Neill, F. Severin, and R. Maev, Can. J. Phys. 82, 981 (2004).

[13] V. A. Shutilov, Basics of Ultrasound Physics (Leningrad University Press, Leningrad, 1980). 
[14] F. D. Tappert and N. J. Zabusky, Phys. Rev. Lett. 27, 1774 (1971).

[15] E. N. Pelinovsky, Prikl. Mekh. Tekh. Fiz. 6, 80 (1971).

[16] R. S. Johnson, J. Fluid Mech. 54, 81 (1972).

[17] V. Djordjevic and L. Redekopp, J. Phys. Oceanogr. 8, 1016 (1978).

[18] R. Grimshaw, E. Pelinovsky, and T. Talipova, Geophys. Astrophys. Fluid Dyn. (to be published).

[19] O. Nakoulima, N. Zahibo, E. Pelinovsky, T. Talipova, and A. Kurkin, Chaos 15, 037107 (2005).

[20] C. C. Mei and Y. Li, Phys. Rev. E 70, 016302 (2004).

[21] R. Grimshaw, E. Pelinovsky, and T. Talipova, Phys. Lett. A 344, 203 (2005).

[22] A. I. Lurie, Nonlinear Theory of Elasticity (Elsevier, Amsterdam, 1990).

[23] F. D. Murnaghan, Finite Deformations of an Elastic Solid (Wiley, New York, 1951).

[24] Mathematica and Wolfram Mathematica are trademarks of Wolfram Research, Inc. www.wolfram.com

[25] A. E. H. Love, A Treatise on the Mathematical Theory of Elasticity (Dover, New York, 1944).

[26] H. N. Abramson, H. J. Plass, and E. A. Ripperger, Adv. Appl. Mech. 5, 111 (1958).

[27] E. I. Grigolyuk and I. T. Selezov, Non-Classical Theories of Vibrations of Rods, Plates and Shells (VINITI, Moscow, 1973)

[28] E. Volterra, Arch. Appl. Mech. 24, 392 (1956).
[29] E. Volterra and E. C. Zachmanoglou, Dynamics of Vibrations (Merrill, Columbus, 1965).

[30] J. W. Miles, J. Fluid Mech. 79, 157 (1977).

[31] G. V. Dreiden, K. R. Khusnutdinova, A. M. Samsonov, and I. V. Semenova, Strain (to be published).

[32] P. G. Drazin, Solitons, London Mathematical Society Lecture Note Series No. 85 (Cambridge University Press, Cambridge, 1983).

[33] G. B. Whitham, Linear and Nonlinear Waves (Wiley, New York, 1999).

[34] L. D. Landau and E. M. Lifshitz, Quantum Mechanics (Pergamon, London, 1959).

[35] A. V. Porubov and A. M. Samsonov, Tech. Phys. Lett. 19, 355 (1993).

[36] A. V. Porubov and G. A. Maugin, Int. J. Non-Linear Mech. 40, 1041 (2005).

[37] T. Kakutani and N. Yamasaki, J. Phys. Soc. Jpn. 45, 674 (1976).

[38] J. Gear and R. Grimshaw, Phys. Fluids 26, 14 (1983).

[39] E. Fermi, J. Pasta, and S. Ulam, Los Alamos Scientific Laboratory Report No. LA-1940, 1955 (unpublished); E. Fermi, J. Pasta, and S. Ulam, Lect. Appl. Math. 15, 143 (1974).

[40] W. Dreyer, M. Herrmann, and J. D. M. Rademacher, Analysis, Modeling and Simulation of Multiscale Problems (Springer, Berlin, 2006), p. 467.

[41] G. A. Maugin, Nonlinear Waves in Elastic Crystals (Oxford University Press, Oxford, 1999). 